\title{
Beauty mesons in lattice QCD with exact chiral symmetry
}

\author{
TWQCD Collaboration \\ Ting-Wai Chiu ${ }^{\text {a,*, }}$, Tung-Han Hsieh ${ }^{\text {b }}$, Chao-Hsi Huang ${ }^{\text {a }}$, Kenji Ogawa ${ }^{\text {a }}$ \\ a Department of Physics and National Center for Theoretical Sciences, National Taiwan University, Taipei 10617, Taiwan \\ ${ }^{\mathrm{b}}$ Research Center for Applied Sciences, Academia Sinica, Taipei 115, Taiwan \\ Received 22 May 2007; accepted 8 June 2007 \\ Available online 12 June 2007 \\ Editor: M. Cvetič
}

\begin{abstract}
We present the first study of treating $\mathbf{b}, \mathbf{c}$, and $\mathbf{s}$ quarks as Dirac fermions in quenched lattice QCD with exact chiral symmetry. For 100 gauge configurations generated with single-plaquette action at $\beta=7.2$ on the $32^{3} \times 60$ lattice, we compute point-to-point quark propagators for 33 quark masses in the range $0.01 \leqslant m_{q} a \leqslant 0.85$, and measure the time-correlation function of (pseudo-)scalar, (pseudo-)vector, and tensor mesons, for symmetric and asymmetric quark masses respectively. The lowest-lying mass spectra of mesons with quark contents $\mathbf{b} \overline{\mathbf{b}}$, $\mathbf{c} \overline{\mathbf{b}}, \mathbf{s} \overline{\mathbf{b}}$, and $\mathbf{c} \overline{\mathbf{c}}$ are determined, together with the pseudoscalar decay constants. Our results are summarized in the tables. Furthermore, we also determine the $\mathbf{b}$ and c quark masses, $m_{b}^{\overline{\mathrm{MS}}}\left(m_{b}\right)=4.65(5) \mathrm{GeV}, m_{c}^{\overline{\mathrm{MS}}}\left(m_{c}\right)=1.16(4) \mathrm{GeV}$.
\end{abstract}

(c) 2007 Elsevier B.V. All rights reserved.

PACS: 11.15.Ha; 11.30.Rd; $12.38 . \mathrm{Gc} ; 14.40 . \mathrm{Lb} ; 14.40 . \mathrm{Nd}$

Keywords: Lattice QCD; Heavy quarks; Charmed mesons; Beauty mesons

\section{Introduction}

Currently, one of the most important themes in high energy physics is to find out whether there is any new physics beyond the Standard Model (SM). In order to identify any discrepancies between the high energy experimental results and theoretical values derived from the SM, the latter have to be obtained in a framework which preserves all crucial features of the SM. Otherwise, it is difficult to determine whether such a discrepancy is due to new physics, or just the approximations (or models) one has used.

So far, the largest uncertainties in the theoretical predictions of the SM stem from the sector of the strong interaction, namely, QCD. Theoretically, lattice QCD is the most viable framework to tackle QCD nonperturbatively from the first principles. However, in practice, any lattice QCD calculation suffers

\footnotetext{
* Corresponding author.

E-mail address: twchiu@phys.ntu.edu.tw (T.-W. Chiu).
}

from the discretization and finite volume errors (which can be systematically improved).

Since all quarks in QCD are excitations of Dirac fermion fields, it is vital to preserve this important feature in any approaches to unveil new physics beyond the SM. Theoretically, the most appealing lattice fermion scheme is the domainwall/overlap fermion [1-3], which preserves the exact chiral symmetry at finite lattice spacing [4], thus provides a proper formulation of QCD on the lattice. However, in practice, it is difficult to accommodate all quark flavors with presently accessible lattice sizes, since the quark masses span at least 3 orders of magnitude (from $m_{u} \sim 5 \mathrm{MeV}$ to $m_{b} \sim 4600 \mathrm{MeV}$ ), even excluding the top quark. In fact, one still cannot put the physical $\mathbf{u} / \mathbf{d}$ quark on a lattice, with sufficiently large number of sites in each direction such that the discretization and finite volume errors are well under control. Thus, one can only perform simulations at unphysically heavy $\mathbf{u} / \mathbf{d}$ quark masses, and then use chiral perturbation theory to extrapolate to the physical limit (i.e., $m_{\pi} \simeq 140 \mathrm{MeV}$ ). This scenario is not expected to have dramatic changes in the next few years. 
Nevertheless, if one only aims at putting $\mathbf{b}, \mathbf{c}$, and $\mathbf{s}$ quarks on the lattice (with $m_{q} a<1$, and $M_{h} L>4$ ), then a lattice of size $\sim 32^{3} \times 60$ (with inverse lattice spacing $a^{-1}=7.68 \mathrm{GeV}$ ) seems to be sufficient for this purpose. As we will see below, the meson mass spectra turn out in good agreement with the experimental values, for quark contents $\mathbf{b} \overline{\mathbf{b}}, \mathbf{c} \overline{\mathbf{b}}, \mathbf{c} \overline{\mathbf{c}}$, and $\mathbf{s} \overline{\mathbf{b}}$.

Note that our approach is fundamentally different from other lattice QCD calculations using relativistic heavy quark formalism, or heavy quark effective theory (HQET), or non-relativistic QCD (NRQCD), in which the systematic errors are difficult to control.

In this Letter, we will restrict ourselves to mesons with quark contents $\mathbf{b} \overline{\mathbf{b}}, \mathbf{c} \overline{\mathbf{b}}, \mathbf{c} \overline{\mathbf{c}}$, and $\mathbf{s} \overline{\mathbf{b}}$. Our results of the masses and decay constants of the pseudoscalar mesons $B_{s}$ and $B_{c}$, and also the masses of the vector mesons $B_{s}^{*}$ and $B_{c}^{*}$, have been presented in Ref. [5]. For the mass spectra of spin- $1 / 2$ and spin-3/2 baryons with quark contents bbb, bbc, bcc, bcs, bss, and ccc, our results will be presented in a forthcoming paper.

\section{Lattice quarks with exact chiral symmetry}

To implement exact chiral symmetry on the lattice [1-4], we consider the optimal domain-wall fermion proposed by Chiu [6, 7]. The action of optimal domain-wall fermion can be written as [7]

$$
\begin{aligned}
\mathcal{A}_{F}= & \sum_{s, s^{\prime}=0}^{N_{s}+1} \sum_{x, x^{\prime}} \bar{\psi}(x, s)\left\{\left(\omega_{s} D_{w}\left(x, x^{\prime}\right)+\delta_{x, x^{\prime}}\right) \delta_{s s^{\prime}}\right. \\
& +\left(\omega_{s} D_{w}\left(x, x^{\prime}\right)-\delta_{x, x^{\prime}}\right) \\
& \left.\times\left(P_{+} \delta_{s^{\prime}, s-1}+P_{-} \delta_{s^{\prime}, s+1}\right)\right\} \psi\left(x^{\prime}, s^{\prime}\right)
\end{aligned}
$$

with boundary conditions

$P_{+} \psi(x,-1)=-r m_{q} P_{+} \psi\left(x, N_{s}+1\right)$,

$P_{-} \psi\left(x, N_{s}+2\right)=-r m_{q} P_{-} \psi(x, 0), \quad r=\frac{1}{2 m_{0}}$,

where $m_{q}$ is the bare quark mass, and the weights $\left\{\omega_{s}, s=\right.$ $\left.1, \ldots, N_{s}\right\}$ are specified by the exact formula derived in Ref. [6] such that the system possesses the maximal chiral symmetry for any fixed $N_{s}$. Here $H_{w}=\gamma_{5} D_{w}$, and $D_{w}$ is the standard Wilson Dirac operator plus a negative parameter $-m_{0}\left(0<m_{0}<2\right)$. The quark fields are constructed from the boundary modes at $s=0$ and $s=N_{s}+1$ with $\omega_{0}=\omega_{N_{s}+1}=0$ [7]:

$$
\begin{aligned}
& q(x)=\sqrt{r}\left[P_{-} \psi(x, 0)+P_{+} \psi\left(x, N_{s}+1\right)\right], \\
& \bar{q}(x)=\sqrt{r}\left[\bar{\psi}(x, 0) P_{+}+\bar{\psi}\left(x, N_{s}+1\right) P_{-}\right] .
\end{aligned}
$$

After introducing pseudofermions with $m_{q}=2 m_{0}$, the generating functional for $n$-point Green's function of the quark fields can be derived as [7],

$$
\begin{aligned}
& Z[J, \bar{J}] \\
& \quad=\frac{\int[d U] e^{-\mathcal{A}_{g}} \operatorname{det}\left[\left(D_{c}+m_{q}\right)\left(1+r D_{c}\right)^{-1}\right] \exp \left\{\bar{J}\left(D_{c}+m_{q}\right)^{-1} J\right\}}{\int[d U] e^{-\mathcal{A}_{g}} \operatorname{det}\left[\left(D_{c}+m_{q}\right)\left(1+r D_{c}\right)^{-1}\right]},
\end{aligned}
$$

where $\mathcal{A}_{g}$ is the action of the gauge fields, $\bar{J}$ and $J$ are the Grassman sources of $q$ and $\bar{q}$ respectively, and

$$
\begin{gathered}
D_{c}=2 m_{0} \frac{1+\gamma_{5} S_{\mathrm{opt}}}{1-\gamma_{5} S_{\mathrm{opt}}}, \\
S_{\mathrm{opt}}=\frac{1-\prod_{s=1}^{N_{s}} T_{s}}{1+\prod_{s=1}^{N_{s}} T_{s}}, \\
T_{s}=\frac{1-\omega_{s} H_{w}}{1+\omega_{s} H_{w}} .
\end{gathered}
$$

From (1), the valence quark propagator in background gauge field is

$\langle q(x) \bar{q}(y)\rangle=-\left.\frac{\delta^{2} Z[J, \bar{J}]}{\delta \bar{J}(x) \delta J(y)}\right|_{J=\bar{J}=0}=\left(D_{c}+m_{q}\right)_{x, y}^{-1}$,

where $D_{c}$ is exactly chirally symmetric $\left(D_{c} \gamma_{5}+\gamma_{5} D_{c}=0\right)$ in the limit $N_{s} \rightarrow \infty$, and its deviation from exact chiral symmetry due to finite $N_{s}$ is the minimal provided that the weights $\left\{\omega_{s}\right\}$ are fixed according to the formula derived in Ref. [6]. Note that in this framework, the bare mass $m_{q}$ (no matter heavy or light) in the valence quark propagator $\left(D_{c}+m_{q}\right)^{-1}$ is well defined for any gauge configurations.

We generate 100 gauge configurations with single plaquette gauge action at $\beta=7.2$ on the $32^{3} \times 60$ lattice. For $m_{0}=1.3$ and $N_{s}=128$, we fix the weights $\left\{\omega_{s}\right\}$ with $\lambda_{\min }=0.1$ and $\lambda_{\max }=6.4$, where $\lambda_{\min } \leqslant \lambda\left(\left|H_{w}\right|\right) \leqslant \lambda_{\max }$ for all gauge configurations. For each configuration, point to point valence quark propagators are computed for 33 bare quark masses in the range $0.01 \leqslant m_{q} a \leqslant 0.85$, with stopping criteria $10^{-11}$ for the conjugate gradient. Then the norm of the residual vector of each column of the quark propagator is less than $2 \times 10^{-11}$

$\left\|\left(D_{c}+m_{q}\right) Y-\mathbb{1}\right\|<2 \times 10^{-11}$,

and the chiral symmetry breaking due to finite $N_{s}$ is less than $10^{-14}$,

$\sigma=\left|\frac{Y^{\dagger} S_{\mathrm{opt}}^{2} Y}{Y^{\dagger} Y}-1\right|<10^{-14}$

In this Letter, we measure the time-correlation function

$C_{\Gamma}(t)=\left\langle\sum_{\vec{x}} \operatorname{tr}\left\{\Gamma\left(D_{c}+m_{Q}\right)_{x, 0}^{-1} \Gamma\left(D_{c}+m_{q}\right)_{0, x}^{-1}\right\}\right\rangle$

for scalar $(S)$, pseudoscalar $(P)$, vector $(V)$, axial-vector $(A)$, and tensor $(T)$ mesons, with Dirac matrix $\Gamma=\left\{\mathbb{1}, \gamma_{5}, \gamma_{i}, \gamma_{5} \gamma_{i}\right.$, $\left.\gamma_{5} \gamma_{4} \gamma_{i}\right\}$ respectively. For vector mesons, we average over $i=$ 1, 2, 3 components, namely,

$C_{V}(t)=\left\langle\frac{1}{3} \sum_{i=1}^{3} \sum_{\vec{x}} \operatorname{tr}\left\{\gamma_{i}\left(D_{c}+m_{Q}\right)_{x, 0}^{-1} \gamma_{i}\left(D_{c}+m_{q}\right)_{0, x}^{-1}\right\}\right\rangle$.

Similarly, we perform the same averaging for axial-vector and tensor mesons.

The time-correlation function $C_{\Gamma}(t)$ is measured for the following three categories:

- Symmetric masses with $m_{Q}=m_{q}$ for 33 quark masses. 
- Asymmetric masses with fixed $m_{Q}=m_{b}=0.68 a^{-1}$, and $m_{q}$ running over all different quark masses.

- Asymmetric masses with fixed $m_{Q}=m_{c}=0.16 a^{-1}$, and $m_{q}$ running over all different quark masses.

\section{Determination of $a^{-1}, m_{b}, m_{c}$, and $m_{s}$}

In Ref. [8], we determine the inverse lattice spacing from the pion decay constant, with experimental input $f_{\pi}=131 \mathrm{MeV}$. However, in this Letter, we do not use the same method since the smallest quark mass turns out to be rather heavy $\left(\simeq m_{s} / 2\right)$, thus chiral extrapolation to $m_{q} \simeq 0$ does not seem to be feasible. Nevertheless, we can use the mass and decay constant of the pseudoscalar meson $\eta_{c}(2980)$ to determine $m_{c}$ and $a^{-1}$ simultaneously. This can be seen as follows.

For symmetric masses $m_{Q}=m_{q}$, the pseudoscalar timecorrelation function $C_{P}(t)\left(\Gamma=\gamma_{5}\right)$ is measured, and fitted to the usual formula

$\frac{z^{2}}{2 m_{P} a}\left[e^{-m_{P} a t}+e^{-m_{P} a(T-t)}\right]$

to extract the mass $m_{P} a$ and the decay constant

$f_{P} a=2 m_{q} a \frac{z}{m_{P}^{2} a^{2}}$.

Then the ratio $m_{P} / f_{P}$ can be obtained for each $m_{q}$.

Since $f_{\eta_{c}}$ has not been measured in HEP experiments, we do not have a physical value for the ratio $m_{\eta_{c}} / f_{\eta_{c}}$. Nevertheless, we can obtain the ratio $m_{\eta_{c}} / f_{\eta_{c}}=6.8(2)$ from our previous study of pseudoscalar mesons on the $20^{3} \times 40$ lattice at $\beta=6.1$ [8]. Then we can use this theoretical value 6.8 to discriminate which $m_{q}$ can give the ratio $m_{P} / f_{P}$ closest to this value. We find that at $m_{q} a=0.16$ the ratio $m_{P} / f_{P}=6.8(1)$, which is the closest to 6.8. Thus we fix $m_{c} a=0.16$. Then we use the experimental mass of $\eta_{c}(2980)$ to determine $a^{-1}$ through the relation

$\left.m_{P} a\right|_{m_{c}}=(2980 \mathrm{MeV}) \times a=0.388(3)$

and obtain $a^{-1}=7680$ (59) $\mathrm{MeV}$. To check the goodness of the values of $m_{c}$ and $a^{-1}$, we compute the time-correlation function of $\overline{\mathbf{c}} \gamma_{i} \mathbf{c}$, and extract the mass of the vector meson equal to 3091(11) MeV, in good agreement with $J / \Psi$ (3097).

The bare mass of strange quark is determined by extracting the mass of vector meson from the time-correlation function $C_{V}(t)$. At $m_{q} a=0.02, m_{V} a=0.1337(5)$, which gives $m_{V}=1027(38) \mathrm{MeV}$, in good agreement with the mass of $\phi(1020)$. Thus we take the strange quark bare mass to be $m_{s} a=0.02$. Similarly, at $m_{q} a=0.68, m_{V} a=1.2308(4)$, which gives $m_{V}=9453(3) \mathrm{MeV}$, in good agreement with the mass of $\Upsilon$ (9460). Thus, we fix the bottom quark bare mass to be $m_{b} a=0.68$.

Note that the spatial size of our lattice $(L \simeq 0.8 \mathrm{fm})$ seems to be small at first glance, however, even for the smallest quark mass $m_{q} a=0.01$, its pseudoscalar mass satisfies $m_{P} L>4$, thus the finite size effects should be well under control.
Table 1

The mass spectra of lowest-lying $(n=1)$ charmonium $\mathbf{\mathbf { c }} \Gamma \mathbf{c}$ states obtained in this work, in comparison with the PDG values in the last column

\begin{tabular}{lllllll}
\hline$\Gamma$ & $J^{P C}$ & $n^{2 S+1} L_{J}$ & {$\left[t_{\min }, t_{\max }\right]$} & $\chi^{2} /$ dof & Mass $(\mathrm{MeV})$ & PDG \\
\hline $\mathbb{1}$ & $0^{++}$ & $1^{3} P_{0}$ & {$[19,42]$} & 0.32 & $3413(14)(9)$ & $\chi_{c 0}(3415)$ \\
$\gamma_{5}$ & $0^{-+}$ & $1{ }^{1} S_{0}$ & {$[22,38]$} & 1.02 & $2980(10)(12)$ & $\eta_{c}(2980)$ \\
$\gamma_{i}$ & $1^{--}$ & $1^{3} S_{1}$ & {$[19,38]$} & 1.18 & $3091(11)(14)$ & $J / \psi(3097)$ \\
$\gamma_{5} \gamma_{i}$ & $1^{++}$ & $1^{3} P_{1}$ & {$[18,42]$} & 0.51 & $3516(13)(8)$ & $\chi_{c 1}(3510)$ \\
$\gamma_{5} \gamma_{4} \gamma_{i}$ & $1^{+-}$ & $1^{1} P_{1}$ & {$[19,43]$} & 0.41 & $3526(13)(9)$ & $h_{c}(3524)$ \\
\hline
\end{tabular}

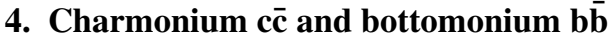

First of all, we check to what extent we can reproduce the charmonium mass spectra which have been measured precisely by high energy experiments.

Our results of the mass spectra of the lowest-lying states of charmonium are summarized in Table 1. The first column is the Dirac matrix used for computing the time-correlation function (2). The second column is $J^{P C}$ of the state. The third column is the conventional spectroscopic notation. The fourth column is the $\left[t_{\min }, t_{\max }\right]$ used for fitting the data of $C_{\Gamma}(t)$ to the usual formula

$\frac{z^{2}}{2 M a}\left[e^{-M a t}+e^{-M a(T-t)}\right]$

to extract the meson mass $M$ and the decay amplitude $z$. The fifth column is the mass $M$ of the state, where the first error is statistical, and the second is our estimate of systematic error based on all fittings satisfying $\chi^{2} /$ dof $<1.3$ and $\left|t_{\max }-t_{\min }\right| \geqslant 6$ with $t_{\min } \geqslant 10$ and $t_{\max } \leqslant 50$. The last column is the corresponding state in high energy experiments, with the PDG mass value [9]. Evidently, our mass spectra of charmonium are in good agreement with the PDG values. Note that our result of the hyperfine splitting $\left({ }^{3} S_{1}-1{ }^{1} S_{0}\right)$ is 111(14)(18) MeV, comparing with the PDG value $118 \mathrm{MeV}$.

For the pseudoscalar $\eta_{c}$, we also obtain its decay constant $f_{\eta_{c}}$ together with its mass, through the decay amplitude $z$ in the equation

$f_{\eta_{c}} a=2 m_{c} a \frac{z}{m_{\eta_{c}}^{2} a^{2}}$.

Our result is

$f_{\eta_{c}}=438 \pm 5 \pm 6 \mathrm{MeV}$,

where the first error is statistical, and the second is our estimate of systematic error based on all fittings satisfying $\chi^{2} /$ dof $<1.3$ and $\left|t_{\max }-t_{\min }\right| \geqslant 6$ with $t_{\min } \geqslant 10$ and $t_{\max } \leqslant 50$. So far, $f_{\eta_{c}}$ has not been determined in high energy experiments.

Next, we turn to the bottomonium (b) $\overline{\mathbf{b}}$ ) states. Our results of the mass spectra of the lowest-lying states of bottomonium are summarized in Table 2.

First, we look at the pseudoscalar $\eta_{b}$. It was first reported by ALEPH Collaboration [13]. However, it has not been confirmed by other HEP experimental groups. Thus we suspect that its mass 9300(20)(20) MeV might not have been determined accurately. It is interesting to see whether the mass of $\eta_{b}$ will turn out to agree with our theoretical value $9380 \pm 10 \mathrm{MeV}$. 
Table 2

The mass spectra of lowest-lying $(n=1)$ bottomonium $\overline{\mathbf{b}} \Gamma \mathbf{b}$ states obtained in this work. The last column is the experimental state we have identified, and its PDG mass value

\begin{tabular}{lllllll}
\hline$\Gamma$ & $J^{P C}$ & $n^{2 S+1} L_{J}$ & {$\left[t_{\min }, t_{\max }\right]$} & $\chi^{2} /$ dof & Mass $(\mathrm{MeV})$ & PDG \\
\hline $\mathbb{1}$ & $0^{++}$ & $1^{3} P_{0}$ & {$[21,39]$} & 0.21 & $9863(15)(8)$ & $\chi_{b 0}(9859)$ \\
$\gamma_{5}$ & $0^{-+}$ & $1^{1} S_{0}$ & {$[27,35]$} & 0.72 & $9383(4)(2)$ & $\eta_{b}(9300) ?$ \\
$\gamma_{i}$ & $1^{--}$ & $1^{3} S_{1}$ & {$[20,39]$} & 1.19 & $9453(3)(2)$ & $\Upsilon(9460)$ \\
$\gamma_{5} \gamma_{i}$ & $1^{++}$ & $1^{3} P_{1}$ & {$[22,38]$} & 0.13 & $9896(20)(8)$ & $\chi_{b 1}(9893)$ \\
$\gamma_{5} \gamma_{4} \gamma_{i}$ & $1^{+-}$ & $1^{1} P_{1}$ & {$[22,38]$} & 0.10 & $9916(22)(8)$ &
\end{tabular}

Besides the mass of $\eta_{b}$, we also determined its decay constant

$f_{\eta_{b}}=801 \pm 7 \pm 5 \mathrm{MeV}$,

where the first error is statistical, and the second is our estimate of systematic error based on all fittings satisfying $\chi^{2} /$ dof $<1.3$ and $\left|t_{\max }-t_{\min }\right| \geqslant 6$ with $t_{\min } \geqslant 10$ and $t_{\max } \leqslant 50$.

Finally, we note that the tensor meson $h_{b}$ has not been observed in high energy experiments, thus our result of its mass $9916 \pm 30 \mathrm{MeV}$ serves as the first prediction from lattice QCD with exact chiral symmetry. Even though it is obtained in the quenched approximation, we suspect that it might provide a reliable prediction of hadron mass spectra, especially for mesons with heavy b quark.

\section{Mesons with quark contents $s \bar{b}$ and $c \bar{b}$}

The decay constants of heavy-light pseudoscalar mesons (e.g., $f_{B}, f_{B_{s}}, f_{D}$, and $f_{D_{s}}$ ) play an important role in extracting the CKM matrix elements which are crucial for testing the Standard Model via the unitarity of CKM matrix. Theoretically, lattice QCD with exact chiral symmetry provides a reliable framework to compute the masses and decay constants of heavy-light pseudoscalar mesons nonperturbatively from the first principles of QCD. Our theoretical predictions of $f_{D}$ and $f_{D_{s}}$ have been presented in Ref. [8], which turn out in good agreement with the recent experimental results $[10,11]$ from CLEO Collaboration. For a recent review of heavy-light pseudoscalar decay constants from lattice QCD, see Ref. [12] and references therein.

Before we present our results of $f_{B_{s}}$ and $m_{B_{s}}$, we recall the basic formulas as follows. In general, the decay constant $f_{P}$ of a heavy-light pseudoscalar meson $P$ is defined as

$\left\langle 0\left|A_{\mu}(0)\right| P(\vec{p})\right\rangle=i p_{\mu} f_{P}$

where $A_{\mu}(x)=\bar{Q}(x) \gamma_{\mu} \gamma_{5} q(x)$ is the axial-vector current. Using the formula

$\partial_{\mu} A_{\mu}=\left(m_{q}+m_{Q}\right) \bar{Q} \gamma_{5} q$

one obtains

$f_{P}=\left(m_{q}+m_{Q}\right) \frac{\left|\left\langle 0\left|\bar{Q} \gamma_{5} q\right| P(\overrightarrow{0})\right\rangle\right|}{m_{P}^{2}}$,

where the pseudoscalar mass $m_{P} a$ and the decay amplitude $z \equiv$ $\left|\left\langle 0\left|\bar{Q} \gamma_{5} q\right| P(\overrightarrow{0})\right\rangle\right|$ can be obtained by fitting the pseudoscalar
Table 3

The mass spectra of lowest-lying $\overline{\mathbf{b}} \Gamma \mathbf{s}$ meson states obtained in this work. The last column is the experimental state we have identified, and its PDG mass value

\begin{tabular}{lllllll}
\hline$\Gamma$ & $J^{P}$ & $n^{2 S+1} L_{J}$ & {$\left[t_{\min }, t_{\max }\right]$} & $\chi^{2} /$ dof & Mass $(\mathrm{MeV})$ & PDG \\
\hline $\mathbb{1}$ & $0^{+}$ & $1^{3} P_{0}$ & {$[20,40]$} & 0.38 & $5852(15)(12)$ & $B_{s J}^{*}(5850)$ \\
$\gamma_{5}$ & $0^{-}$ & $1^{1} S_{0}$ & {$[26,32]$} & 0.56 & $5385(27)(17)$ & $B_{S}(5368)$ \\
$\gamma_{i}$ & $1^{-}$ & $1^{3} S_{1}$ & {$[25,33]$} & 0.50 & $5424(28)(19)$ & $B_{s}^{*}(5412)$ \\
$\gamma_{5} \gamma_{i}$ & $1^{+}$ & $1^{3} P_{1}$ & {$[18,42]$} & 0.68 & $5884(16)(13)$ & \\
$\gamma_{5} \gamma_{4} \gamma_{i}$ & $1^{+}$ & $1^{1} P_{1}$ & {$[18,38]$} & 0.62 & $5897(16)(12)$ & \\
\hline
\end{tabular}

time-correlation function $C_{P}(t)$ to the usual formula

$\frac{z^{2}}{2 m_{P} a}\left[e^{-m_{P} a t}+e^{-m_{P} a(T-t)}\right]$.

Our results for $B_{s}$ are:

$m_{B_{s}}=5385 \pm 27 \pm 17 \mathrm{MeV}$,

$f_{B_{s}}=253 \pm 8 \pm 7 \mathrm{MeV}$,

where the first error is statistical, and the second is our estimate of systematic error based on all fittings satisfying $\chi^{2} /$ dof $<1.3$ and $\left|t_{\max }-t_{\min }\right| \geqslant 6$ with $t_{\min } \geqslant 10$ and $t_{\max } \leqslant 50$. Our result of $m_{B_{s}}$ is in good agreement with the experimental value $5368 \mathrm{MeV}$ compiled by PDG. Since $f_{B_{s}}$ has not been measured in high energy experiments, our result serves as the first prediction from lattice QCD with exact chiral symmetry.

Next, we present our results of the mass spectra of the lowest-lying states of beauty mesons with quark content $\mathbf{s} \overline{\mathbf{b}}$, which are summarized in Table 3. Here we have identified the scalar $\overline{\mathbf{b}} \mathbf{s}$ meson with the state $B_{S J}^{*}(5850)$ observed in HEP experiments, due to the proximity of their masses. Theoretically, this implies that $B_{s J}^{*}(5850)$ possesses $J^{P}=0^{+}$, which can be verified by HEP experiments in the future. Moreover, we have obtained the masses of the axial-vector and tensor mesons which have not been observed in HEP experiments, as a prediction from lattice QCD.

Next, we turn to the heavy mesons with beauty and charm. For the pseudoscalar $B_{c}$, we obtain

$m_{B_{c}}=6278 \pm 6 \pm 4 \mathrm{MeV}, \quad f_{B_{c}}=489 \pm 4 \pm 3 \mathrm{MeV}$,

where the first error is statistical, and the second is our estimate of systematic error based on all fittings satisfying $\chi^{2} /$ dof $<1.3$ and $\left|t_{\max }-t_{\min }\right| \geqslant 6$ with $t_{\min } \geqslant 10$ and $t_{\max } \leqslant 50$. Our result of $m_{B_{c}}$ is in good agreement with the experimental value 6286(5) $\mathrm{MeV}$ measured by CDF Collaboration [14], while $f_{B_{C}}$ has not been measured by HEP experiments. In principle, $f_{B_{c}}$ can be measured from the leptonic decay $B_{c}^{+} \rightarrow l^{+} v_{l}$, since its decay width is proportional to $f_{B_{c}}^{2}\left|V_{c b}\right|^{2}$.

In Table 4, we summarize our results of the mass spectra of the lowest-lying states of mesons with beauty and charm. Except for the pseudoscalar $B_{c}$, other states have not been observed in experiments. It will be interesting to see to what extent the experimental results would agree with our theoretical values. 
Table 4

The mass spectra of lowest-lying $\overline{\mathbf{b}} \Gamma \mathbf{c}$ meson states obtained in this work. The last column is the experimental state we have identified, and its PDG mass value

\begin{tabular}{lllllll}
\hline$\Gamma$ & $J^{P}$ & $n^{2 S+1} L_{J}$ & {$\left[t_{\min }, t_{\max }\right]$} & $\chi^{2} /$ dof & Mass $(\mathrm{MeV})$ & PDG \\
\hline $\mathbb{1}$ & $0^{+}$ & $1{ }^{3} P_{0}$ & {$[19,41]$} & 0.26 & $6732(13)(9)$ & \\
$\gamma_{5}$ & $0^{-}$ & $1{ }^{1} S_{0}$ & {$[19,38]$} & 1.14 & $6278(6)(4)$ & $B_{c}(6286)$ \\
$\gamma_{i}$ & $1^{-}$ & $1^{3} S_{1}$ & {$[19,38]$} & 1.31 & $6315(6)(5)$ & \\
$\gamma_{5} \gamma_{i}$ & $1^{+}$ & $1{ }^{3} P_{1}$ & {$[19,41]$} & 0.27 & $6778(12)(7)$ & \\
$\gamma_{5} \gamma_{4} \gamma_{i}$ & $1^{+}$ & $1^{1} P_{1}$ & {$[18,42]$} & 0.45 & $6796(10)(7)$ & \\
\hline
\end{tabular}

\section{Masses of $b$ and $c$ quarks}

In the Standard Model, the quark masses are fundamental parameters which have to be determined from high energy experiments. However, they cannot be measured directly since quarks are confined inside hadrons, unlike an isolated electron whose mass and charge both can be measured directly from its responses in electric and magnetic fields. Therefore, the quark masses can only be determined by comparing a theoretical calculation of physical observables (e.g., hadron masses) with the experimental values. Evidently, for any field theoretic calculation, the quark masses depend on the regularization, as well as the renormalization scheme and scale. One of the objectives of lattice QCD is to compute the hadron masses nonperturbatively from the first principles, and from which the quark masses are determined.

We have used the mass of the vector meson $\Upsilon(9460)$ to fix the bare mass of $\mathbf{b}$ quark equal to $m_{b}=0.68 a^{-1}=$ 5.22(4) GeV. ${ }^{1}$ To transcribe the bare mass to the corresponding value in the usual renormalization scheme $\overline{\mathrm{MS}}$ in high energy phenomenology, one needs to compute the lattice renormalization constant $Z_{m}=Z_{s}^{-1}$, where $Z_{s}$ is the renormalization constant for $\bar{\psi} \psi$. In general, $Z_{m}$ should be determined nonperturbatively. However, in this case, the lattice spacing is rather small $(a \simeq 0.026 \mathrm{fm}$ ), thus we suspect that the one-loop perturbation formula [15]

$Z_{s}(\mu)=1+\frac{g^{2}}{4 \pi^{2}}\left[\ln \left(a^{2} \mu^{2}\right)+0.17154\right] \quad\left(m_{0}=1.30\right)$

already provides a very good approximation for $Z_{s}$. At $\beta=$ $7.2, a^{-1}=7.680(59) \mathrm{GeV}$, and $\mu=2 \mathrm{GeV}$, (7) gives $Z_{s}=$ 1.086(2), which transcribes the bare mass $m_{b}=5.22(4) \mathrm{GeV}$ to

$m_{b}^{\overline{\mathrm{MS}}}(2 \mathrm{GeV})=4.81 \pm 0.04 \mathrm{GeV}$,

where the error is due to the uncertainty in the lattice spacing. Now if we also include the uncertainty in the determination of the $\mathbf{b}$ quark bare mass, which is estimated to be $\delta\left(m_{b} a\right)=0.002$, then we obtain

$m_{b}^{\overline{\mathrm{MS}}}(2 \mathrm{GeV})=4.81 \pm 0.05 \mathrm{GeV}$.

In order to compare our result with the PDG average, we have to obtain $m_{b}^{\overline{\mathrm{MS}}}$ at the scale $\mu=m_{b}$. This can be obtained

\footnotetext{
1 Note that we have not tuned the value of $m_{b}$, thus the mass of $\Upsilon$ is 9453(3)(2) MeV (see Table 2) rather than $9460 \mathrm{MeV}$.
}

Table 5

The decay constants of pseudoscalar mesons obtained in this work, together with their masses. They are identified with the corresponding PDG mesons listed in the last column, however, the decay constants have not been measured in HEP experiments

\begin{tabular}{llllll}
\hline$\overline{\mathbf{Q}} \Gamma q$ & {$\left[t_{\min }, t_{\max }\right]$} & $\chi^{2} /$ dof & Mass $(\mathrm{MeV})$ & $f_{P}(\mathrm{MeV})$ & PDG \\
\hline$\overline{\mathbf{b}} \gamma_{5} \mathbf{b}$ & {$[27,35]$} & 0.72 & $9383(4)(2)$ & $801(7)(5)$ & $\eta_{b}(9300)$ \\
$\overline{\mathbf{b}} \gamma_{5} \mathbf{c}$ & {$[19,38]$} & 1.14 & $6278(6)(4)$ & $489(4)(3)$ & $B_{c}(6287)$ \\
$\overline{\mathbf{b}} \gamma_{5} \mathbf{s}$ & {$[26,32]$} & 0.56 & $5385(27)(17)$ & $253(8)(7)$ & $B_{S}(5368)$ \\
$\overline{\mathbf{c}} \gamma_{5} \mathbf{c}$ & {$[22,38]$} & 1.02 & $2980(10)(12)$ & $438(5)(6)$ & $\eta_{c}(2980)$ \\
\hline
\end{tabular}

by solving the equation $\bar{m}_{b}=m_{b} Z_{m}\left(\mu=\bar{m}_{b}\right)$. Our result is

$m_{b}^{\overline{\mathrm{MS}}}\left(m_{b}\right)=4.65 \pm 0.05 \mathrm{GeV}$

which seems to be higher than the PDG average $4.20 \pm 0.07$ [9].

Now we turn to the c quark mass. Using (7), the c quark bare mass $m_{c}=0.160(5) a^{-1}$ is transcribed to

$m_{c}^{\overline{\mathrm{MS}}}(2 \mathrm{GeV})=1.13 \pm 0.04 \mathrm{GeV}$,

where the error incorporates the uncertainties in the lattice spacing and the $\mathbf{c}$ quark bare mass. Again, to compare our result with the PDG average, we obtain $m_{c}^{\overline{\mathrm{MS}}}$ at the scale $\mu=m_{c}$,

$m_{c}^{\overline{\mathrm{MS}}}\left(m_{c}\right)=1.16 \pm 0.04 \mathrm{GeV}$

which is in good agreement with the PDG average $1.25 \pm 0.09$ [9].

\section{Concluding remark}

We have performed the first study of treating $\mathbf{b}$, $\mathbf{c}$, and $\mathbf{s}$ quarks as Dirac fermions in lattice QCD with exact chiral symmetry. The lowest-lying mass spectra of mesons with quark contents $\mathbf{b} \overline{\mathbf{b}}, \mathbf{c} \overline{\mathbf{b}}, \mathbf{s} \overline{\mathbf{b}}$, and $\mathbf{c} \overline{\mathbf{c}}$ are determined, together with the pseudoscalar decay constants. Our results of the meson mass spectra in Tables 1-4, pseudoscalar decay constants in Table 5, and the $\mathbf{b}$ and $\mathbf{c}$ quark masses in (9) and (11), suggest that lattice QCD with exact chiral symmetry is a viable framework to study heavy quark physics from the first principles of QCD.

For systems involving $\mathbf{u} / \mathbf{d}$ quarks, one may use several quark masses in the range $m_{u / d}<m_{q}<m_{s}$ to perform the chiral extrapolation. To this end, one may choose a coarser lattice (e.g. $\beta=7.0$ ), then it is possible to accommodate a wide range of quark masses $m_{s} / 4<m_{q} \leqslant m_{b}$ on the $42^{3} \times 64$ lattice, without significant discretization and finite-size errors. This study is now in progress. Evidently, it has become feasible to treat heavy and light quarks as Dirac fermions on the lattice, in lattice QCD with exact chiral symmetry.

\section{Acknowledgements}

This work was supported in part by the National Science Council, Republic of China, under the Grant No. NSC95-2112M002-005 (T.W.C.), and Grant No. NSC95-2112-M001-072 (T.H.H.), and by the National Center for High Performance Computation, and the Computer Center at National Taiwan University. 


\section{References}

[1] D.B. Kaplan, Phys. Lett. B 288 (1992) 342;

D.B. Kaplan, Nucl. Phys. B (Proc. Suppl.) 30 (1993) 597.

[2] R. Narayanan, H. Neuberger, Nucl. Phys. B 443 (1995) 305.

[3] H. Neuberger, Phys. Lett. B 417 (1998) 141.

[4] P.H. Ginsparg, K.G. Wilson, Phys. Rev. D 25 (1982) 2649.

[5] T.W. Chiu, T.H. Hsieh, TWQCD Collaboration, PoS LAT2006 (2007) 180.

[6] T.W. Chiu, Phys. Rev. Lett. 90 (2003) 071601.

[7] T.W. Chiu, hep-lat/0303008;

T.W. Chiu, Nucl. Phys. B (Proc. Suppl.) 129 (2004) 135.
[8] T.W. Chiu, T.H. Hsieh, J.Y. Lee, P.H. Liu, H.J. Chang, Phys. Lett. B 624 (2005) 31.

[9] W.M. Yao, et al., Particle Data Group, J. Phys. G 33 (2006) 1.

[10] M. Artuso, et al., CLEO Collaboration, Phys. Rev. Lett. 95 (2005) 251801.

[11] M. Artuso, et al., CLEO Collaboration, hep-ex/0704.0629.

[12] T. Onogi, PoS LAT2006 (2007) 017.

[13] A. Heister, et al., ALEPH Collaboration, Phys. Lett. B 530 (2002) 56.

[14] A. Abulencia, et al., CDF Collaboration, Phys. Rev. Lett. 96 (2006) 082002.

[15] C. Alexandrou, E. Follana, H. Panagopoulos, E. Vicari, Nucl. Phys. B 580 (2000) 394 